\title{
Autologous Mesenchymal Stem Cells Show More Benefit on Systolic Function Compared to Bone Marrow Mononuclear Cells in a Porcine Model of Chronic Myocardial Infarction
}

\author{
T. I. G. van der $\operatorname{Spoel}^{1}$ • W. A. Gathier ${ }^{1} \cdot$ S. Koudstaal ${ }^{1}$ • F. van Slochteren ${ }^{1} \cdot$ S. Jansen of \\ Lorkeers $^{1}$ - J. P. G. Sluijter ${ }^{1,2}$ • I. E. Hoefer ${ }^{3}$ • P. Steendijk ${ }^{4}$ - M. J. M. Cramer ${ }^{1}$. \\ P. A. Doevendans ${ }^{1,2} \cdot$ E. van Belle ${ }^{1}$. S. A. J. Chamuleau ${ }^{1}$ \\ Received: 9 January 2015 / Accepted: 6 July 2015 / Published online: 17 September 2015 \\ (C) The Author(s) 2015. This article is published with open access at Springerlink.com
}

\begin{abstract}
Cardiac cell therapy is a strategy to treat patients with chronic myocardial infarction (MI). No consensus exists regarding the optimal cell type. First, a comparison between autologous bone marrow-derived mononuclear cells (BMMNC) and mesenchymal stem cells (MSC) on therapeutic efficacy after MI was performed. Next, the effect of repetitive, NOGA-guided transendocardial injection was determined via a crossover design. Nineteen pigs were allocated in three groups: (1) placebo (at 4 and 8 weeks), (2) MSC (followed by placebo at 8 weeks), or (3) BMMNC (followed by MSC at 8 weeks) delivery including a priming strategy to enhance MSC effect. At 4 weeks, ejection fraction (EF) was significantly improved after MSC injection and not by BMMNC injection. After 8 weeks, no difference was observed in EF between cell-treated groups demonstrating the positive systolic effect of MSC. This study showed that MSC rather than BMMNC injection improves systolic function in chronic MI.
\end{abstract}

Associate Editor Enrique Lara-Pezzi oversaw the review of this article

S. A. J. Chamuleau

s.a.j.chamuleau@umcutrecht.nl

1 Department of Cardiology, Division Heart and Lungs, University Medical Center Utrecht, Rm E03.511, Heidelberglaan 100, 3584 CX Utrecht, The Netherlands

2 Interuniversity Cardiology Institute of the Netherlands (ICIN), Utrecht, The Netherlands

3 Department of Experimental Cardiology, Utrecht, The Netherlands

4 Department of Cardiology, Leiden University Medical Center, Leiden, The Netherlands
Keywords Stem cells - Ischemic cardiomyopathy · Mesenchymal stem cell $\cdot$ Systolic function

\section{Introduction}

Ischemic heart failure remains a major cause of morbidity and mortality [1]. Stem cell therapy emerged as an innovative and attractive therapeutic approach for patients with chronic myocardial infarction (MI). The ultimate goal of this treatment is to support and enhance the endogenous repair mechanisms by replacing dysfunctional cardiomyocytes and inducing angiogenesis.

In clinical and preclinical studies, a modest improvement in left ventricular ejection fraction (LVEF) was observed using a single injection of bone marrow cells after MI $[2,3]$. Our preclinical meta-analysis showed that the choice of cell type is an important significant predictor of improvement in LVEF [3] suggesting a trend towards more pronounced effects of mesenchymal stem cells (MSC). Till now, bone marrow mononuclear cells (BMMNC) and MSC have been well studied in patients with ischemic heart disease [4]. However, it is known that functional differences between MSC and BMMNC exist [3]. A direct comparison on functional endpoints between these cell types has not been performed so far. We hypothesized that pretreatment of the area of interest could be helpful to further enhance the effects of MSC. Thus, we incorporated a repetitive cell injection strategy in the study design.

Percutaneous transendocardial (TE) delivery, guided by electromechanical mapping (NOGA), was shown to be safe in patients with chronic ischemic cardiomyopathy [5] and has the advantage of detecting hibernating myocardium which is the area that will probably profit most from cell delivery [6]. 
Our objective was to determine the most potent regenerative strategy using autologous bone marrow cell types, i.e., BMMNC and MSC, in a large animal model of ischemia/ reperfusion injury. First, a direct comparison between BMMNC and MSC was performed 4 weeks after transplantation. Second, the effect of repetitive injection after initial priming was determined by including a crossover with MSC in the BMMNC group with an additional follow-up period of 4 weeks.

\section{Method}

\section{Animals}

Nineteen female Dutch Landrace pigs received humane care in compliance with the "Guide for the Care and Use of Laboratory Animals," published by the National Institutes of Health (National Institutes of Health publication 85-23, revised 1985). The study protocol was approved by the Animal Experimentation Committee of the University of Utrecht.

\section{Study Design}

Animals were allocated to one of three groups: (group 1) placebo (phosphate buffered saline (PBS), Invitrogen, Carlsbad, CA, USA), (group 2) $10^{7}$ autologous MSC, or (group 3) $10^{7}$ autologous BMMNC injection at 4 weeks. Eight weeks after MI (thus, 4 weeks after initial injection), the animals in group 3 received an additional injection of MSC to determine whether priming could rescue the damaged myocardium, while the other groups received an injection with PBS for control purposes. Twelve weeks after the initial MI, the animals were euthanized and tissue was prepared for histology. Cardiac function was assessed by pressure-volume (PV) loops and echocardiography. The study design is shown in Fig. 1.

\section{Premedication and Anesthesia}

After an overnight fast, animals were sedated with an intramuscular injection of ketamin $(10 \mathrm{mg} / \mathrm{kg})$, midazolam $(0.5 \mathrm{mg} / \mathrm{kg})$, and atropin $(0.04 \mathrm{mg} / \mathrm{kg})$. Next, thiopental $(4 \mathrm{mg} / \mathrm{kg}$ ) was administered intravenously before intubation. They were intubated with an endotracheal tube and anesthetized in the supine position. The animals were mechanically ventilated with the use of a positive pressure ventilator with a mix of oxygen and air (FiO2 0.5). General anesthesia/ analgesia was maintained with midazolam $(0.5 \mathrm{mg} / \mathrm{kg} / \mathrm{h}$, Roche, Woerden, the Netherlands), sufentanyl citrate $(2 \mu \mathrm{g} /$ $\mathrm{kg} / \mathrm{h}$, Janssen-Cilag, Tilburg, the Netherlands), and pancuronium bromid $(0.1 \mathrm{mg} / \mathrm{kg} / \mathrm{h}$, Organon, Oss, the Netherlands). Metoprolol (Centrafarm, Etten-Leur, the Netherlands) was administered intravenously ( $5 \mathrm{mg}$ ) to reduce the mechanical irritation of the heart. During surgery, animals were anticoagulated with heparin $(\mathrm{ACT}>250 \mathrm{~s})$. At the end of the experiment, the animals were euthanized by pentobarbital overdose.

\section{Myocardial Ischemia/Reperfusion Model}

During the entire procedure, electrocardiogram, arterial pressure, and capnogram were continuously monitored. Prior to MI, all animals received an oral dose of amiodarone $(400 \mathrm{mg}$ / day; starting 10 days prior to MI) and clopidogrel (75 mg/day; starting 3 days prior to MI; Sanofi Aventis, Gouda, the Netherlands) [7]. A bolus of $500 \mathrm{mg}$ acetylic salicylic acid (Centrafarm, Etten-Leur, the Netherlands) was given the day before the occlusion. Myocardial infarction was created by a percutaneous balloon of equivalent size to the proximal left circumflex artery (LCX). The balloon was inflated for $75 \mathrm{~min}$ at 5-8 atm [8]. Complete occlusion of the LCX was confirmed by angiography. To prevent ventricular arrhythmias, $300 \mathrm{mg}$ amiodarone (Centrafarm, Etten-Leur, the Netherlands) intravenously was given. External defibrillation (150-200 J) was used when ventricular fibrillation occurred. After the procedure, coronary angiography was performed to confirm vessel patency. After recovery, the animals received daily an oral dose of $50 \mathrm{mg}$ metoprolol, $400 \mathrm{mg}$ amiodarone, $75 \mathrm{mg}$ clopidogrel, and $160 \mathrm{mg}$ acetylic salicylic acid until termination to prevent thrombosis and arrhythmias [7].

\section{MSC Culture and Labeling}

Bone marrow was aspirated $(35-40 \mathrm{~mL})$ from the sternum by a heparinized syringe. BMMNC were isolated by Ficoll density gradient centrifugation and frozen in $10 \%$ DMSO and $90 \%$ culture medium.

MSC were isolated and characterized as previously described [9]. Autologous MSC were cultured at $37^{\circ} \mathrm{C}$ in Alpha MEM (Invitrogen, Carlsbad, CA, USA), supplemented with $10 \%$ FBS, heparin, and $1 \%$ penicillin/streptomyocin. Cells were cultured, replacing medium every 3 days and used between passage 5 and 7. Before injection, cells were resuspended in $2 \mathrm{~mL}$ PBS and viability was assessed via trypan-blue (Sigma-Aldrich, St. Louis, MO, USA) counting.

\section{Transendocardial Delivery}

To enable TE injection, an 8-F sheath was placed in a carotid artery. Next, a mapping catheter (Biosense Webster, Cordis, Johnson \& Johnson, USA) was placed retrogradely through the aortic valve into the left ventricle (LV). First, a 3dimensional electromechanical map of the LV was obtained using the NOGA system (Biosense Webster, Cordis, Johnson \& Johnson, USA), as described before [10, 11]. Hereafter, 10 injections of $0.2 \mathrm{~mL}$ were slowly placed using the 
Fig. 1 Study design. $B M M N C$ bone marrow mononuclear cells, Echo echocardiography, $M I$ myocardial infarction, $M S C$ mesenchymal stem cells, $P$ V loop pressure-volume loop

$\begin{array}{cccc}\text { MI } & 1^{\text {st }} \text { Injection } & 2^{\text {nd }} \text { Injection } & \text { Sacrifice } \\ -4 \text { weeks } & \mathrm{t}=0 & 4 \mathrm{wk} & 8 \mathrm{wk}\end{array}$

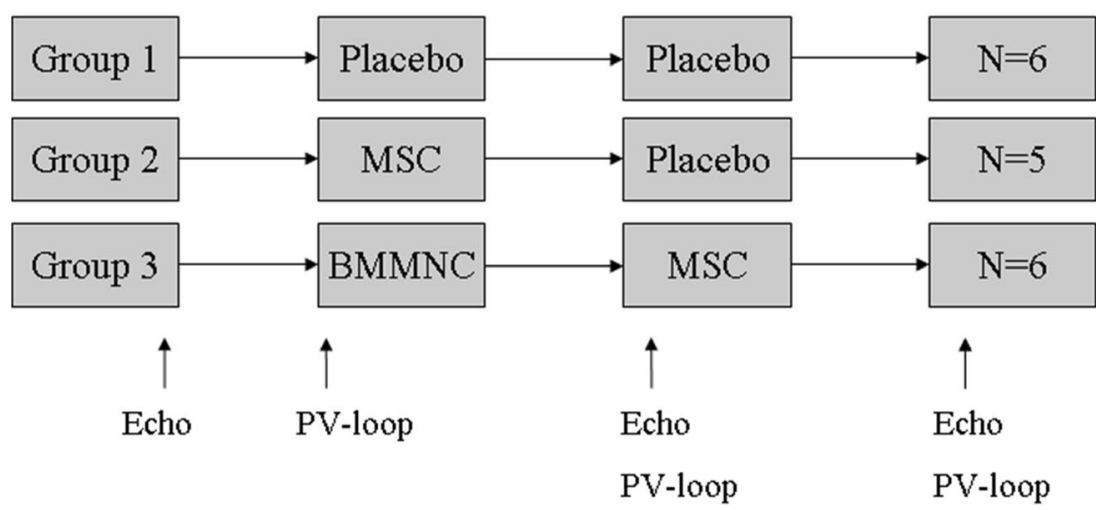

Histology
MYOSTAR ${ }^{\circledR}$ injection catheter (Biosense Webster, Cordis, Johnson \& Johnson, Diamond Bar, USA). Two injections were placed in the infarct zone and eight in the border zone. Four weeks after the first injection, this procedure was repeated and the second injections were given at the same location. Injections were only given in areas with a unipolar voltage greater than $6 \mathrm{mV}[10,11]$.

\section{Echocardiography}

A transthoracic echocardiogram (5-MHz probe, IE-33, Philips, Best, the Netherlands) was performed directly after MI, 8 weeks after MI, and at sacrifice as described before [7]. Short axis images were obtained at the papillary level, and three consecutive cardiac cycles were acquired. Wall thickness (WT) of the posterolateral wall was assessed in end-systole and end-diastole. The left ventricular internal area (LVIA) was obtained without including the papillary muscles in end-systole and end-diastole. The fractional area shortening was calculated as ((LVIAendiastole-LVIAendsystole)/ LVIAenddiastole $) \times 100$.

\section{Pressure-Volume Loop protocol}

Pressure-volume loops were obtained using a 7-F conductance catheter that was inserted via a carotid artery and placed along the long axis of the LV. The catheter was connected with a signal processor (Leycom CFL, CD Leycom, Zoetermeer, the Netherlands). The correct position of the conductance catheter was verified by angiography and by inspection of the segmental conductance signals. The conductance signals were calibrated by thermodilution and hypertonic saline dilution via a 7-F Swan-Ganz catheter that was placed into the right or left pulmonary artery $[12,13]$. Data were collected during steady-state conditions with the respirator systems turned off at end-expiration. From these signals, hemodynamic indices were derived. Data analysis and calculations were performed using custom-made software (CD Leycom, Zoetermeer, the Netherlands), as previously described [14]. Parameters of global systolic and diastolic function were calculated during steady-state conditions at 4, 8, and 12 weeks after MI. Cardiac output (CO) measured by Swan-Ganz was corrected by multiplying each measurement with 0.62 . This number was based on the following equation (CO Swan-Ganz at sacrifice/ $\mathrm{CO}$ transonic aorta flow probe at sacrifice). The isovolumic relaxation time constant (Tau) was calculated by phase-plot analysis. The end-systolic pressure-volume relationship was measured by its slope end-systolic elastance (Ees). Diastolic stiffness (Eed) was determined as the lineair slope of the enddiastolic pressure-volume relationship. Both were calculated by single-beat analysis as described earlier [15].

\section{Histology}

After euthanasia, the LV was weighed and tissue samples from the infarct, borderzone, and remote region of the heart were obtained. Samples were fixed in $4 \%$ formalin at room temperature. Before cutting 5- $\mu \mathrm{m}$ sections, samples were embedded in paraffin for analysis. For quantification of collagen content, picrosirius red staining and detection with circularly polarized light and digital image microscopy was used [16]. Five random images at $\times 20$ magnification of the infarcted, 
borderzone, and remote area were obtained per animal. After conversion into gray value images, the average number of grey values was expressed as a mean grey value per square micrometer. Capillary density was assessed by Lectin staining (Sigma-Aldrich) and counterstained with Hematoxilin and Eosin to identify nuclei. In total, five fields per section at $\times 20$ magnification were counted per animal per zone.

\section{Statistical Analysis}

Values derived from echocardiography were analyzed in a blinded fashion. For statistical analysis, we used a linear mixed effects model to account for repeated measurements on each animal. In this model, we included a generalized estimation equations-type matrix to account for the association between residual covariance, e.g., time point of measurement ( 8 and 12 weeks after MI). Statistical comparison of data between groups was done using a one-way ANOVA with a post hoc Tukey or Kruskal-Wallis test. Data are presented as mean $\pm \mathrm{SE}$ or median with interquartile ranges in case of nonnormal distributed data. All statistical analyses were performed using SPSS 18.1.1, and $P$ values $<0.05$ were considered statistically significant.

\section{Results}

\section{Procedural Data}

In total, 19 animals underwent the MI procedure. One animal in the placebo died due to severe heart failure evidenced by obduction (group 1; day 71 after MI), and one animal had to be terminated for reaching a human-defined endpoint due to an abscess at the right foot not related to the study (group 2). MSC viability (group 2, $92 \pm 4 \%$ vs. group 3, $93 \pm 1 \% ; P=$ 0.10 ) and number of MSC (group 2, $1.0 \pm 0.1 \times 10^{7}$ vs. group 3, $\left.0.9 \pm 0.2 \times 10^{7} ; P=0.10\right)$ did not differ between the cell-treated groups. BMMNC viability was $92 \pm 4 \%$ and the injected number $1.7 \pm 0.2 \times 10^{7}$. No cardiac tamponade or sustained ventricular arrhythmias were observed after any cell or placebo injection.

Table 1 Hemodynamics derived from pressure-volume loops at baseline, before the second injection and at sacrifice

\begin{tabular}{|c|c|c|c|c|c|c|c|c|c|}
\hline \multirow{2}{*}{$\begin{array}{l}\text { Hemodynamics } \\
\text { Parameter }\end{array}$} & \multicolumn{3}{|c|}{ Baseline (4 weeks after MI) } & \multicolumn{3}{|c|}{ Injection ( 8 weeks after MI) } & \multicolumn{3}{|c|}{ Sacrifice (12 weeks after MI) } \\
\hline & Group 1 & Group 2 & Group 3 & Group 1 & Group 2 & Group 3 & Group 1 & Group 2 & Group 3 \\
\hline \multicolumn{10}{|l|}{ General } \\
\hline Weight (kg) & $73 \pm 3$ & $71 \pm 2$ & $73 \pm 1$ & $80 \pm 3$ & $76 \pm 2$ & $76 \pm 1$ & $83 \pm 3$ & $82 \pm 3$ & $82 \pm 1$ \\
\hline LV weight (g) & & & & & & & $168 \pm 7$ & $159 \pm 5$ & $175 \pm 9$ \\
\hline FAS $(\%)$ & & & & $50 \pm 5$ & $55 \pm 3$ & $47 \pm 2$ & $50 \pm 2$ & $51 \pm 2$ & $43 \pm 3$ \\
\hline HR (beats/min) & $52 \pm 2$ & $59 \pm 4$ & $52 \pm 6$ & $63 \pm 8$ & $56 \pm 1$ & $57 \pm 7$ & $51 \pm 7$ & $55 \pm 1$ & $53 \pm 4$ \\
\hline $\mathrm{CO}(\mathrm{L} / \mathrm{min})$ & $3.5 \pm 0.3$ & $2.8 \pm 0.2$ & $3.0 \pm 0.4$ & $3.2 \pm 0.2$ & $3.5 \pm 0.3^{\S}$ & $2.7 \pm 0.2$ & $2.8 \pm 0.5$ & $3.5 \pm 0.3$ & $3.1 \pm 0.1$ \\
\hline \multicolumn{10}{|l|}{ Systole } \\
\hline ESV (mL) & $41 \pm 3$ & $37 \pm 4$ & $49 \pm 7$ & $50 \pm 12$ & $31 \pm 8$ & $44 \pm 7$ & $51 \pm 11$ & $23 \pm 5^{\#}$ & $30 \pm 6^{*}$ \\
\hline ESP $(\mathrm{mmHg})$ & $96 \pm 7$ & $87 \pm 11$ & $100 \pm 5$ & $86 \pm 7$ & $90 \pm 8$ & $85 \pm 4$ & $91 \pm 7$ & $81 \pm 5$ & $74 \pm 4 *$ \\
\hline $\mathrm{EF}(\%)$ & $62 \pm 2$ & $57 \pm 2$ & $55 \pm 5$ & $54 \pm 7$ & $69 \pm 3^{\# \S}$ & $54 \pm 5$ & $52 \pm 3$ & $74 \pm 3^{\#}$ & $69 \pm 4^{*}$ \\
\hline $\mathrm{dP} / \mathrm{dt}_{\mathrm{MAX}}(\mathrm{mmHg} / \mathrm{s})$ & $1586 \pm 131$ & $1390 \pm 208$ & $1374 \pm 46$ & $1372 \pm 152$ & $1351 \pm 134$ & $1096 \pm 64$ & $1460 \pm 102$ & $1402 \pm 40^{\S}$ & $1033 \pm 71^{*}$ \\
\hline Ees $(\mathrm{mmHg} / \mathrm{ml})$ & $3.9 \pm 0.5$ & $4.2 \pm 0.6$ & $3.7 \pm 0.1$ & $3.7 \pm 0.7$ & $3.7 \pm 0.4$ & $3.2 \pm 0.2$ & $4.1 \pm 0.9$ & $3.7 \pm 0.5$ & $2.5 \pm 0.3 *$ \\
\hline \multicolumn{10}{|l|}{ Diastole } \\
\hline EDV (mL) & $107 \pm 8$ & $85 \pm 5$ & $109 \pm 6$ & $106 \pm 13$ & $92 \pm 13$ & $95 \pm 9$ & $106 \pm 16$ & $86 \pm 9$ & $91 \pm 10$ \\
\hline EDP (mmHg) & $16 \pm 1$ & $13 \pm 1$ & $16 \pm 1$ & $14 \pm 2$ & $16 \pm 1$ & $13 \pm 2$ & $15 \pm 1$ & $14 \pm 2$ & $11 \pm 1 * \S$ \\
\hline $\mathrm{dP} / \mathrm{dt}_{\mathrm{MIN}}(\mathrm{mmHg} / \mathrm{s})$ & $-1428 \pm 131$ & $-1345 \pm 165$ & $-1393 \pm 91$ & $-1350 \pm 224$ & $-1447 \pm 119$ & $-1275 \pm 99$ & $-1239 \pm 224$ & $-1328 \pm 99$ & $-1148 \pm 93$ \\
\hline PHT (ms) & $34 \pm 2$ & $31 \pm 2$ & $39 \pm 3$ & $36 \pm 7$ & $31 \pm 1$ & $34 \pm 3$ & $44 \pm 7$ & $28 \pm 1^{\#}$ & $31 \pm 1^{*}$ \\
\hline Tau (ms) & $58 \pm 4$ & $52 \pm 4$ & $67 \pm 6$ & $62 \pm 14$ & $51 \pm 2$ & $57 \pm 5$ & $72 \pm 16$ & $48 \pm 2$ & $49 \pm 2 *$ \\
\hline Eed $(\mathrm{mmHg} / \mathrm{mL})$ & $0.38 \pm 0.04$ & $0.42 \pm 0.02$ & $0.37 \pm 0.06$ & $0.46 \pm 0.8^{*}$ & $0.54 \pm 0.06^{\S}$ & $0.29 \pm 0.02$ & $0.30 \pm 0.04$ & $0.38 \pm 0.07$ & $0.24 \pm 0.03$ \\
\hline
\end{tabular}

Data are presented as mean $\pm \mathrm{SE}$

$C O$ cardiac output, $H R$ heart rate, $E D P$ end-diastolic pressure, $E S P$ end-systolic pressure, $d P / d t_{M A X}$ maximal rate of $L V$ pressure increase, $d P / d t_{M I N}$ minimal rate of LV pressure decrease, $E D V$ end-diastolic volume, Eed myocardial stiffness, Ees end-systolic elastance, $E S V$ end-systolic volume, $E F$ ejection fraction, $F A S$ fractional area shortening, $L V$ left ventricle, $P H T$ pressure halftime, Tau isovolumic relaxation time constant

${ }^{*} P<0.05$ BMMNC vs. placebo, ${ }^{\#} P<0.01 \mathrm{MSC}$ vs. placebo, ${ }^{\S} P<0.05 \mathrm{MSC}$ vs. BMMNC 


\section{Comparison Between MSC and BMMNC on Cardiac Function at 4 weeks after Cell Transplantation}

Four weeks after MI (baseline), no difference in LVEF between groups was observed $(P=0.30$; Table 1$)$. When comparing LVEF differences between baseline and 4 weeks after injection (Fig. 2), placebo-treated animals showed a reduction in LVEF whereas in MSC-treated animals, LVEF was significantly improved (group 2, $11.9 \pm 3 \%$ vs. group 1, $-7.8 \pm 8 \%$; $P=0.002$ ). Animals treated with MSC showed a tendency for having a decrease in $\triangle \mathrm{ESV}$ (group $2,-6.0 \pm 7 \mathrm{~mL}$ vs. group 1 , $10 \pm 10 \mathrm{~mL} ; P=0.10)$. No significant difference in $\triangle \mathrm{LVEF}$ between BMMNC and placebo treatment was observed (group $3,-1.6 \pm 6 \%$ vs. group $1,-7.8 \pm 8 \% ; P=0.748$ ). Consequently, MSC injection led to a significant increase in $\triangle \mathrm{LVEF}$ compared to BMMNC injection (group 2, 11.9 $\pm 3 \%$ vs. group $3,-1.6 \pm 6 \% ; P=0.028)$ but also significantly improved $\Delta \mathrm{CO}$ (group $2,0.7 \pm 0.3 \mathrm{~L} / \mathrm{min}$ vs. group $3,-0.4 \pm$ $0.4 \mathrm{~L} / \mathrm{min} ; P=0.037$ ) and thereby reflects an increased systolic cardiac performance. After BMMNC injection, a trend for impaired $\Delta \mathrm{dP} / \mathrm{dt}_{\mathrm{MAX}}$ was observed compared to MSCtreated animals (group 2, $-38 \pm 154 \mathrm{mmHg}$ vs. group 3, $-277 \pm 57 \mathrm{mmHg} ; P=0.08)$.

Regarding global diastolic function, no significant difference in $\Delta$ end-diastolic volume between groups could be observed (group 1, $-0.2 \pm 4 \mathrm{~mL}$; group 2, 7.7 $\pm 13 \mathrm{~mL}$; group 3, $-14 \pm 8 \mathrm{~mL}$; all $P>0.1$ ). In addition, $\mathrm{dP} / \mathrm{dt}_{\mathrm{MIN}}$, Tau, enddiastolic pressure (EDP), and pressure halftime (PHT) were similar in the different treatment groups (Table 1). However, passive diastolic function was improved in the BMMNC group compared to the other groups, indicated by $\Delta$ Eed (group
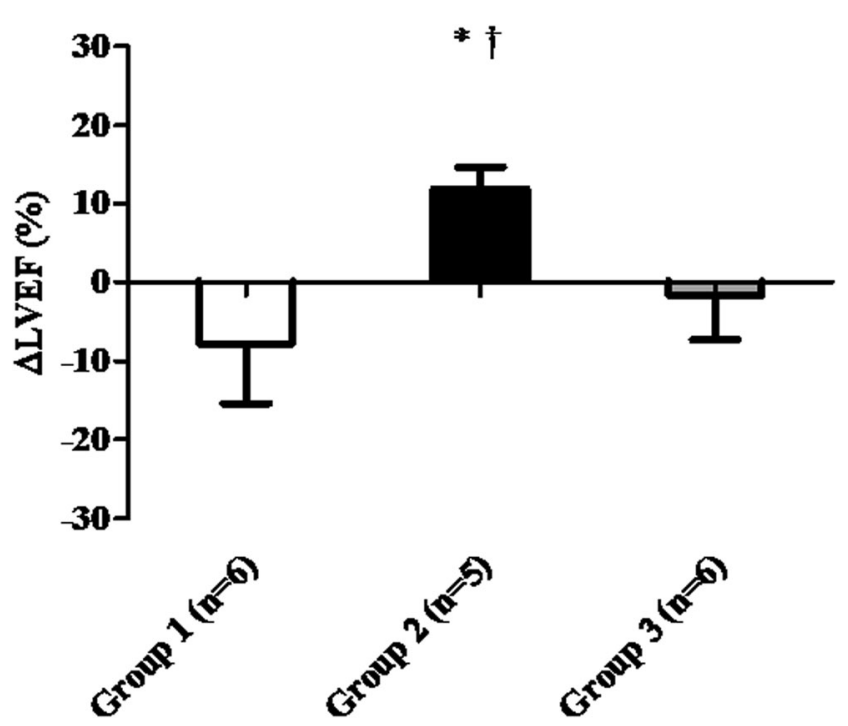

Fig. 2 Effects at 4 weeks after cell therapy: group 2 (MSC injection) improves systolic function compared to group 3 (BMMNC) and group 1 (placebo). Percentage of change in LVEF between baseline and 4 weeks after injection in each treatment group. ${ }^{*} P<0.01$ compared to group 1 . $\dagger P=0.028$ compared to group 3. $L V E F$ left ventricular ejection fraction§
1, $0.08 \pm 0.05 \mathrm{mmHg} / \mathrm{mL}$; group 2, $0.12 \pm 0.08 \mathrm{mmHg} / \mathrm{mL}$; group 3, $-0.08 \pm 0.05 \mathrm{mmHg} / \mathrm{mL}$; BMMNC vs. placebo, $P=$ 0.04 ; MSC vs. BMMNC, $P=0.004$; $\mathrm{MSC}$ vs. placebo, $P=$ 0.349 ).

Directly after MI, echocardiographic recordings showed that end-systolic WT was similar between groups (group 1, $1.25 \pm 0.2 \mathrm{~cm}$; group $2,1.38 \pm 0.1 \mathrm{~cm}$; group $3,1.01 \pm 0.2 \mathrm{~cm}$; $P=0.78)$. Also, no difference in end-diastolic WT was observed (group 1, $1.21 \pm 0.3 \mathrm{~cm}$; group 2, $1.18 \pm 0.3 \mathrm{~cm}$; group $3,1.01 \pm 0.2 \mathrm{~cm} ; P=0.48)$. Four weeks after treatment, no significant effect on $\Delta$ end-diastolic WT (group 1, $0.03 \pm$ $0.06 \mathrm{~cm}$; group 2, $0.01 \pm 0.03 \mathrm{~cm}$; group $30.10 \pm 0.05 \mathrm{~cm}$ ) and $\Delta$ end-systolic WT (group 1, $0.28 \pm 0.07 \mathrm{~cm}$; group 2, $0.06 \pm 0.06 \mathrm{~cm}$; group $3,0.16 \pm 0.09 \mathrm{~cm}$ ) was found.

\section{Effect of Repeated Cell Injection on Cardiac Function at 8 weeks after Cell Transplantation}

Since no effect of BMMNC on $\triangle \mathrm{LVEF}$ was observed, we now did not expect a synergistic effect of repetitive BMMNC injection in group 3. However, we now were able to study whether a second injection of MSC could rescue the damaged myocardium.

When comparing $\triangle \mathrm{LVEF}$ between baseline and at sacrifice (Fig. 3), placebo-treated animals showed a reduction in $\triangle \mathrm{LVEF}$, whereas in cell-treated animals, $\triangle \mathrm{EF}$ was significantly improved (group 2, 18 $\pm 3 \%$; group 3, $13 \pm 4 \%$ vs. group 1 , $-9 \pm 3 \%$; all $P<0.01$ ) caused by a significant reduction in
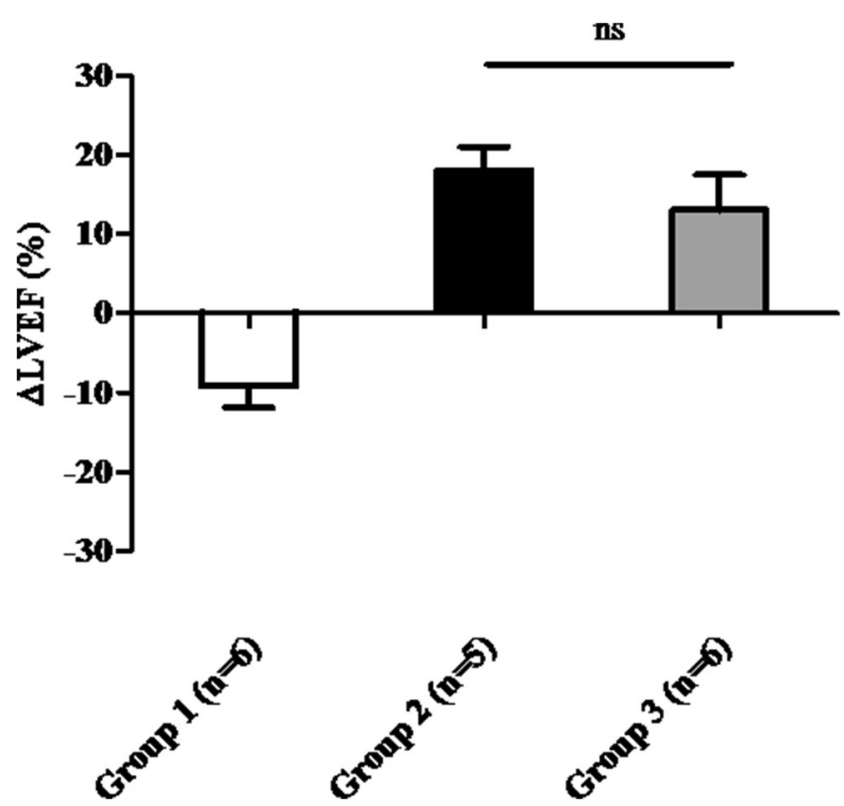

Fig. 3 Effect at 8 weeks after (repeated) cell therapy: no difference on EF between single (group 2) versus pretreated injections (group 3) with MSC. No significant effect on $\triangle \mathrm{LVEF}$ (baseline and 8 weeks after injection) between single and repeated cell injection was observed. $L V E F$ left ventricular ejection fraction 
$\triangle \mathrm{ESV}$ (group 2, $-14 \pm 4 \mathrm{~mL}$; group $3,-20 \pm 4 \mathrm{~mL}$ vs. group 1, $11 \pm 10 \mathrm{~mL}$; all $P<0.01)$. However, no difference in $\triangle \mathrm{EF}$ or $\triangle \mathrm{ESV}$ between single MSC injection and repeated cell delivery could be observed $(P=0.28$ and $P=0.79)$. Contractility measured by $\Delta \mathrm{dP} / \mathrm{dt}_{\mathrm{MAX}}$ was significantly increased after single MSC injection, compared to BMMNC and MSC injection (group 2, 105 $\pm 193 \mathrm{mmHg}$; group 3, $-340 \pm 63 \mathrm{~mL} ; P=0.003$ ). In fact, the second MSC injection on top of the first BMMNC injection (without significant difference compared to placebo) once more revealed the magnitude of effect on systolic function by MSC.

Overall, both cell groups showed an improvement in diastolic active relaxation parameters compared to placebotreated animals. This was reflected by a shortened $\Delta$ Tau and decreased $\triangle \mathrm{PHT}$. Myocardial stiffness (Eed) was unaffected by cell therapy. No statistical difference in active and passive diastolic function between the cell-treated groups could be observed, except for EDP.

No significant difference in echocardiographic parameters between single cell injection and repeated cell injection was observed.

\section{Capillary Density and Collagen}

Histological samples were not available for two animals (both group 1). Due to technical issues, in 175 of the 225 samples (78 \%), representative Lectin stainings were obtained and used for analysis. Both collagen and vascular density data showed a non-normal distribution.

A significantly higher number of capillaries in the infarcted area was seen in group 3 compared to both group 1 and group 2 (median value 104 vs. 36 vs. 57, respectively; $P<0.01$,
Fig. 4). Between groups, no significant difference between the number of vessels in the border zones was found (median value for group 1, 157; group 2, 207; group 3, 209). Also, no difference was found for the remote areas. Within groups, the number of vessels was lower in the infarcted area as compared to border zone and remote area as expected. Furthermore, no significant difference was found in the number of vessels in the remote and border zone. Figure 5 shows representative Lectin-stained images from each group.

Collagen density assessment could be performed on $100 \%$ (225 samples) of the picrosirius red stainings. As expected, infarcted tissue from all groups showed a substantial increase in collagen density compared to tissue from remote areas and border zones.

Group 3 showed a significantly lower collagen density in the infarcted area compared to group 1 (median $64 \times 10^{-7} \mathrm{vs}$. $318 \times 10^{-7}$, respectively; $P<0.01$, Fig. 6), but not group 2 (median $288 \times 10^{-7}$ ). However, a significantly higher collagen density was observed in the border zone of group 3 compared to group 2 (median $29 \times 10^{-7}$ vs. $3 \times 10^{-7}$, respectively; $P<0.05$, Fig. 6). Figure 7 shows representative picrosirius red-stained images from each group.

\section{Discussion}

In this study, we performed a comparison between MSC and BMMNC via TE cell delivery in a porcine model of chronic ischemic heart disease. The main novel findings of our study are the following: (1) MSC are superior to BMMNC in improving systolic function, and (2) the delivery strategy of repeated cell injection was safe and feasible. Interestingly, MSC
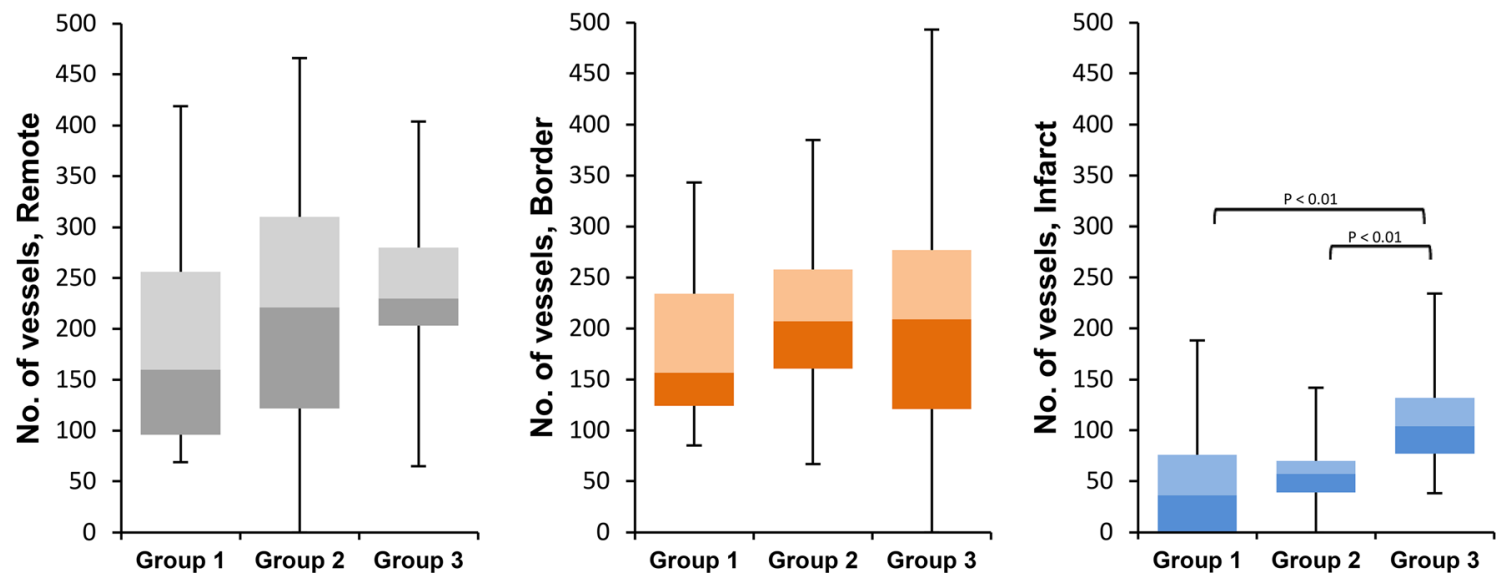

Fig. 4 Microcirculatory remodeling in the damaged myocardium after cell delivery 12 weeks post-MI. Microvascular formation determined by Lectin staining at sacrifice was significantly higher in the infarcted zone in group 3 (BMMNC+MSC injection) compared to the other groups $(P<0.01)$. Furthermore, a nonsignificant increase in vessel density was observed in both cell-treated groups in both the remote and border zones

(both $P>0.1$ ). Group 1: 4 animals, 13 images used for analysis of remote zone, 14 for border zone, 13 for infarct zone. Group 2: 5 animals, 14 images used for analysis of border zone, 23 images for border zone, 18 for infarct zone. Group 3: 6 animals, 23 images used for analysis of remote zone, 27 for border zone, 30 for infarct zone 


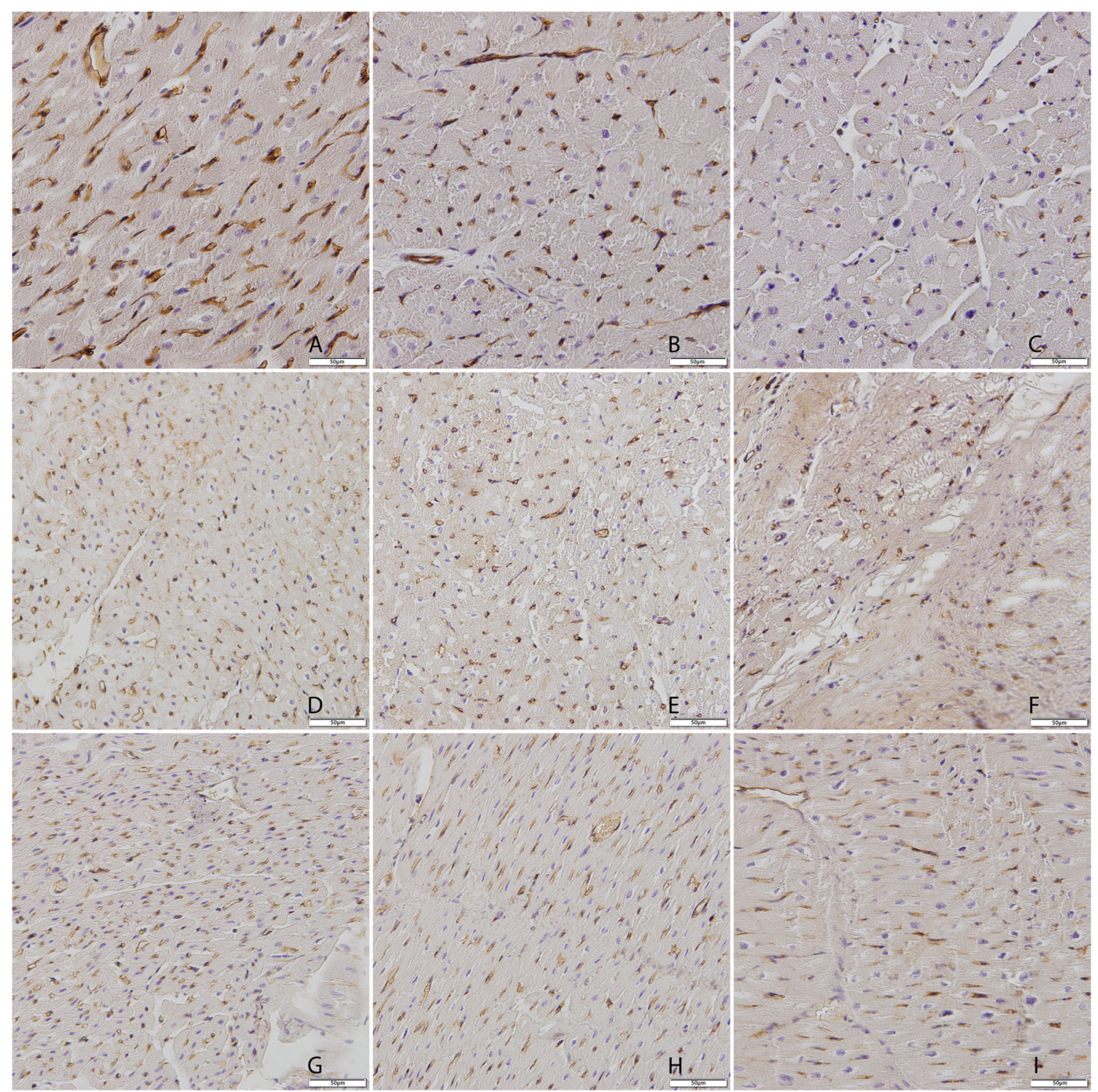

Fig. 5 Representative images of Lectin staining at sacrifice from remote area, border zone, and infarcted tissue. a Group 1 (placebo+placebo) remote, $\mathbf{b}$ group 1 border, $\mathbf{c}$ group 1 infarct. $\mathbf{d}$ Group 2 (MSC+placebo) remote, e group 2 border, f group 2 infarct. g Group 3 (BMMNC+MSC) remote, $\mathbf{h}$ group 3 border, $\mathbf{i}$ group 3 infarct. Magnification $\times 20$ on top of BMMNC led to normalization of LV function, supporting the notion that MSC rather than BMMNC improve systolic function.

\section{MSC Treatment Improves Systolic Function in Contrast to BMMNC}

We performed a head-to-head comparison of treatment with autologous BMMNC and MSC and demonstrated a beneficial effect for MSC on systolic function (EF $11.9 \pm 3 \%$ ), whereas no effect of BMMNC on LVEF was found compared to placebo (EF $-1.6 \pm 6 \%$ and $-7.8 \pm 8 \%$, respectively).

This was despite the fact that even a slightly higher number of cells were used in the BMMNC group $\left(1.7 \times 10^{7} \mathrm{BMMNC}\right.$ vs. $1.0 \times 10^{7} \mathrm{MSC}$ ). This observation is in line with the results of our large preclinical meta-analysis, showing more benefit of MSC in ischemic heart disease compared to BMMNC [3]. On the contrary, Li et al. did not found significant difference between MSC and BMMNC. However, they infused far more BMMNC than MSC (BMMNC $4.7 \pm 1.7 \times 10^{7}$ vs. MSC $6.2 \pm$ $\left.1.6 \times 10^{5}\right)$ [17]. It is known that the number of cells is related to the magnitude of effect $[3,4]$. In our study, no statistical difference between injected cell number was observed. Our results may appear to be in contrast with the data from previous clinical studies that did show modest but significant improvements of LVEF after treatment with BMMNC (approximately 3-5\%) [18-20]. However, such studies were mainly performed in the setting of acute MI and these effects were 


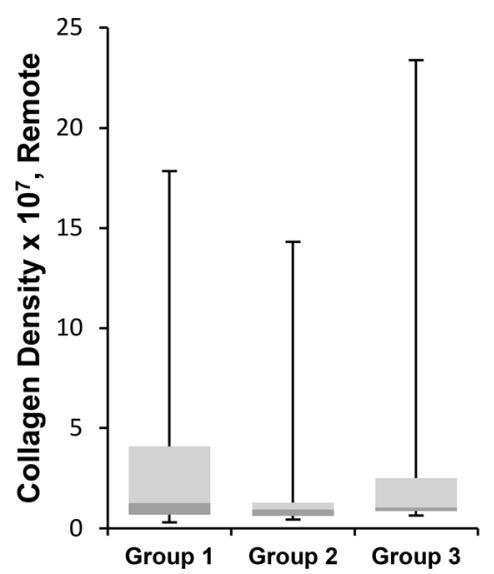

Fig. 6 Collagen density after cell therapy. Collagen quantification 12 weeks post infarction of remote areas, border areas, and infarcted areas. A significantly decreased collagen density was observed in the infarcted zone in group 3 (BMMNC+MSC) compared to group 1 (placebo+placebo) $(P<0.01)$. However, in the border zone of group 3 ,
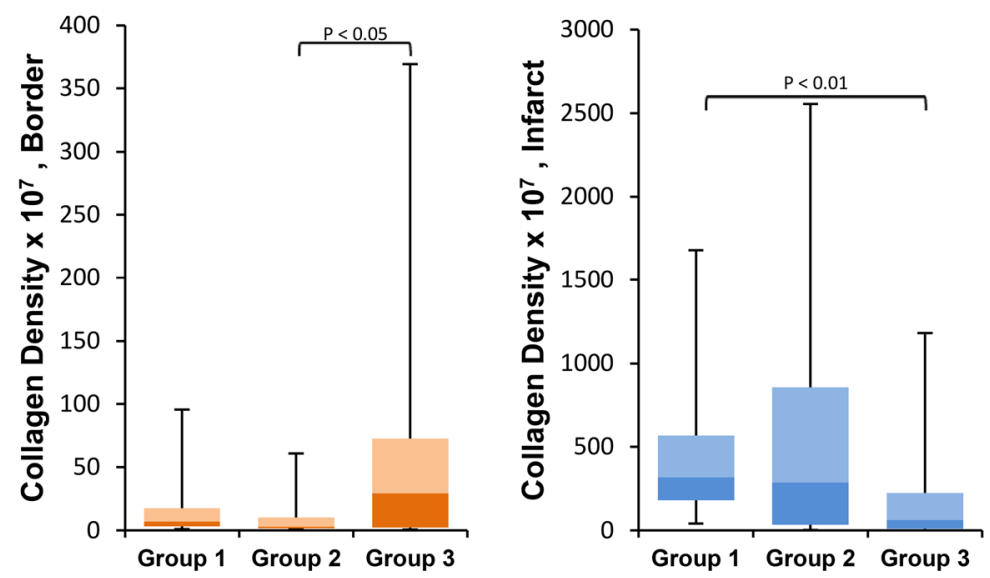

an increase in interstitial fibrosis was measured compared to group 2 (MSC + placebo) $(P<0.05)$. Group 1: 4 animals, 20 images used for analysis per section. Group 2: 5 animals, 25 images used for analysis per section. Group 3: 6 animals, 30 images used for analysis per section. Note differences in $Y$-axis predominantly found in subgroups of large infarctions (baseline $\mathrm{EF}<48 \%$ ) [18]. In fact, several trials with BMMNC in chronic patients did not show improvement of systolic function $[5,21,22]$. On the contrary, in a comparable patient cohort, it was demonstrated that indeed MSC were able to improve cardiac function [23]. Recently, a clinical study (TACHFT trail) directly compared these cells and demonstrated the safety of both cell types [24]. However, after MSC injection, a reduction in infarct size was observed which was not the case after BMMNC. Unfortunately, this study was not powered to provide a definitive statement on therapeutic efficacy. Taken together, these results provide a robust rationale for a larger trial comparing both cell types to determine whether or not bone marrow stem cells have a clinical future.

\section{Repeated Cell Injection was Safe but Does Not Further Improve Cardiac Function}

Repetitive cell injections led to no serious adverse events (e.g., death, persistent ventricular arrhythmias) but did not further improve systolic function compared to single MSC injection. This is largely due to the lack of an effect of BMMNC; this was surprising and not anticipated. Several studies investigated in particular the effect of repetitive cell transplantations [25]. Our observations are in line with a clinical trial investigating the effect of repeated BMMNC injections in patients with chronic heart failure showing no additional benefit of repeated BMMNC treatment on LVEF. [26] However, Yao et al. demonstrated that repeated BMMNC injection in patients with large acute MI resulted in a significant improvement in $\triangle \mathrm{LVEF}$ compared to single cell injection [27]. This effect may be explained by the low baseline LVEF values (20$39 \%$ ) which were higher in our study. Our results are in line with an observation [28], in which skeletal myoblasts were sequential injected in a chronic infarcted porcine myocardium. Although a different cell type was used, repeated cell injections showed no difference in $\triangle \mathrm{LVEF}$ (repeated $15.1 \%$ vs. single $11.1 \%$ ).

\section{Histological Effects of MSC Injection}

It is postulated that a decreased collagen density and an increase in vascular density in border and/or infarct zone could be the explanation for the observed effects on systolic function in groups 2 and 3. Indeed, collagen density was significantly reduced in the infarcted area in group 3 (BMMNC+MSC) compared to group 1 (placebo+placebo). However, this was not the case for group 2. Surprisingly, the opposite was found for the border zone of group 3. Therefore, the histological analysis of infarcted tissue only partly explains the found effects in the present study.

Second, measurement of capillary density showed a significant increase in the number of microvessels in the infarcted area after BMMNC+MSC injection compared to placebo treatment and MSC+placebo treatment. These data do not directly explain the functional changes although there seems to be a slight trend of increased capillary density in both the border zone and infarcted area of both cell groups; however, a significant increase in the number of vessels was only found in the infarcted area of the BMMNC-primed group as seen in Fig. 4. This suggests a more pronounced angiogenic response after priming with BMMNC before injecting MSC (group 3).

Finally, some MSC were observed in the infarcted tissue by fluorescent microscopy (data not shown), but it is unlikely that the observed effect was caused by differentiation of MSC into cardiac lineages as also suggested by others [29]. However, 


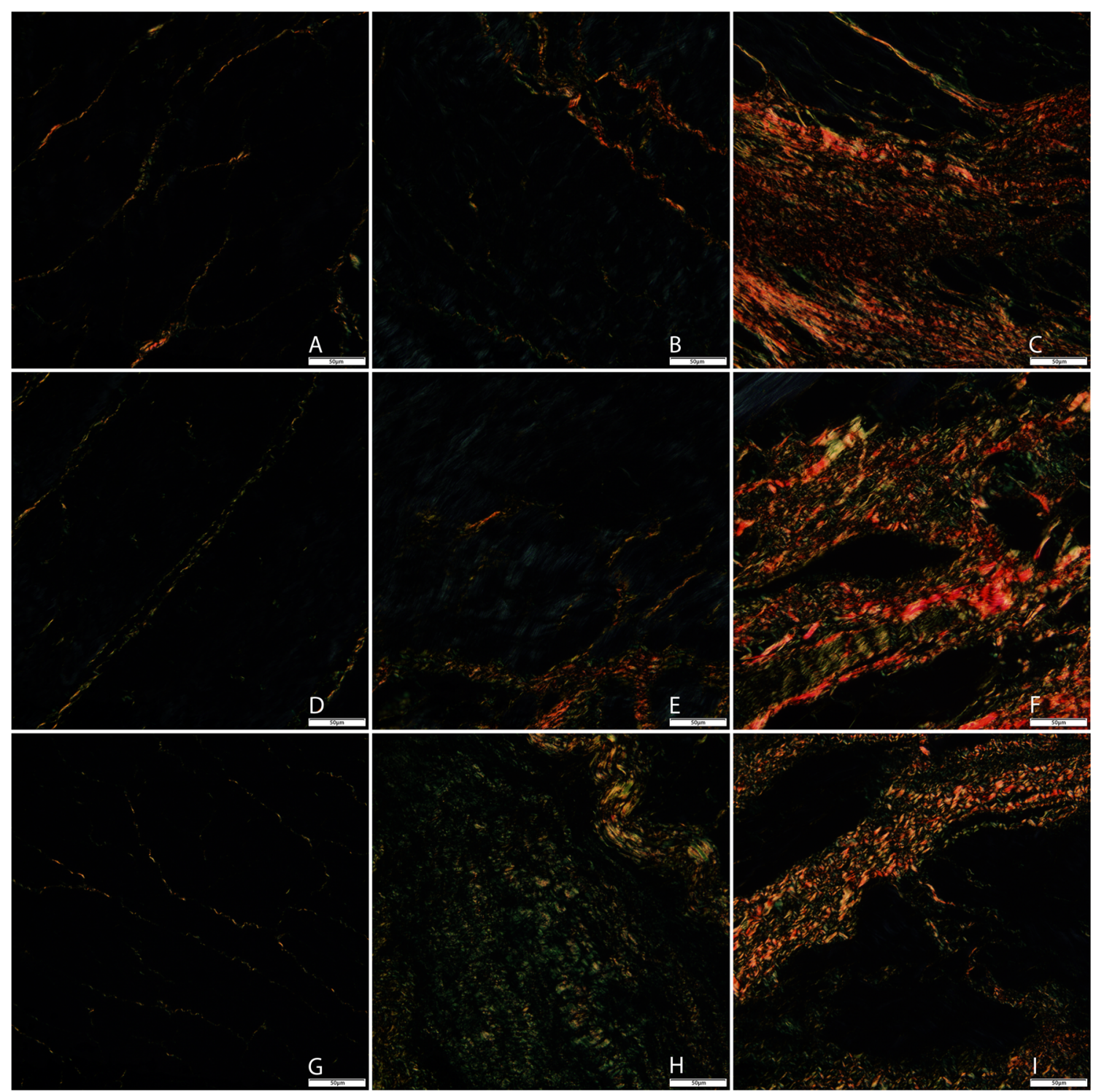

Fig. 7 Representative images of picrosirius red staining at sacrifice from remote area, border zone, and infarcted tissue. a Group 1 (placebo+ placebo) remote, $\mathbf{b}$ group 1 border, $\mathbf{c}$ group 1 infarct, $\mathbf{d}$ group 2 (MSC+

MSC may lead to prolonged secretion of paracrine factors activating capillary angiogenesis.

\section{Study Limitations}

Our ischemia/reperfusion model resulted in a limited decrease in LVEF (appr. $50 \%$ ), but not severe heart failure. This is related to the chosen model (temporary occlusion of LCX for $75 \mathrm{~min}$ ) and maybe by the fact that animals were treated with similar medication protocols (e.g., beta blockers, which may be cardioprotective) compared to the patients suffering from MI. Nevertheless, significant effects on LVEF were observed.

The porcine model is considered the best possible model to resemble the clinical situation, although major differences placebo) remote, e group 2 border, $\mathbf{f}$ group 2 infarct, g group 3 (BMMNC + MSC) remote, $\mathbf{h}$ group 3 border, $\mathbf{i}$ group 3 infarct. Magnification $\times 20$

exist (e.g., risk factors, cell isolation protocols [30], comorbidity, follow-up duration), which prevent direct extrapolation to patient management. Nevertheless, our group demonstrated that large animal models can accurately predict human clinical outcome and these models are frequently used for translational purposes [3].

Care was taken to perform adequate analysis of collagen and vascular density; however, both sampling error and staining issues may have had impact on this histological assessment. By analysing five sections per sample, we attempted to minimize this bias.

Nowadays, cardiac MRI is considered the gold standard to measure LVEF and volumes. However, due to practical reasons, we performed echocardiographic and pressure-volume loop analysis. These techniques are still considered reliable, 
reproducible, and a valid measure of LV function and are therefore most often used in preclinical research models.

Finally, our results show that the anticipated synergistic effect by pretreatment with BMMNC before MSC treatment in group 3 was suggested by the increased capillary density, but was not represented in functional endpoints. Knowing this, a fourth group comparing repetitive MSC injections would have been helpful to elucidate this effect for repetitive MSC treatment. Nevertheless, we feel that the take-home message from this particular group is now reassuring that indeed MSC outperform BMMNC in this setting.

\section{Conclusions}

We demonstrated that MSC are more potent in terms of improvement of LVEF than BMMNC in a chronic model for ischemic heart disease. Our data do not support strategies using repetitive injections, although using different combinations of cells may be of value in more severe heart failure. These data should encourage researchers and clinicians to focus future studies on other cell types (i.e., MSC) than BMMNC.

Acknowledgments We gratefully acknowledge Cees Verlaan, Merel Schurink, Marlijn Jansen, Maringa Emons, and Joyce Visser for excellent technical assistance and animal care. In addition, the authors acknowledge the technical assistance provided by Esther van Eeuwijk and Sridevi Jaksani.

\section{Compliance with Ethical Standards}

Sources of Funding This work was supported by the Netherlands Heart Foundation "[2003B07304 and 2010T025]," BSIK program "Dutch Program for Tissue Engineering" "[grant 6746]," and a Bekalis price (PD). This research is part of the Project P1.04 SMARTCARE of the research program of the BioMedical Materials institute, co-funded by the Dutch Ministry of Economic Affairs, and the CVON (Cardiovascular Research Netherlands) HUSTCARE consortium (SC, PD, WG).

Conflict of Interest The authors declare they have no competing interests.

Ethical Approval All applicable international, national, and/or institutional guidelines for the care and use of animals were followed. This study was performed in compliance with the "Guide for the Care and Use of Laboratory Animals," published by the National Institutes of Health (National Institutes of Health publication 85-23, revised 1985). The study protocol was approved by the Animal Experimentation Committee of the University of Utrecht.

Open Access This article is distributed under the terms of the Creative Commons Attribution 4.0 International License (http:// creativecommons.org/licenses/by/4.0/), which permits unrestricted use, distribution, and reproduction in any medium, provided you give appropriate credit to the original author(s) and the source, provide a link to the Creative Commons license, and indicate if changes were made.

\section{References}

1. Rosamond, W., Flegal, K., Furie, K., et al. (2008). Heart disease and stroke statistics - 2008 update: a report from the American Heart Association Statistics Committee and Stroke Statistics Subcommittee. Circulation, 117, e25-e146. doi:10.1161/ CIRCULATIONAHA.107.187998.

2. Abdel-Latif, A., Bolli, R., Tleyjeh, I. M., et al. (2007). Adult bone marrow-derived cells for cardiac repair: a systematic review and meta-analysis. Archives of Internal Medicine, 167, 989-997. doi: 10.1001/archinte.167.10.989.

3. van der Spoel, T. I. G., of Lorkeers, S. J., Agostoni, P., et al. (2011). Human relevance of pre-clinical studies in stem cell therapy; systematic review and meta-analysis of large animal models of ischemic heart disease. Cardiovascular Research, 91, 649-658. doi:10. 1093/cvr/cvr113.

4. Jeevanantham, V., Butler, M., Saad, A., Abdel-Latif, A., ZubaSurma, E. K., \& Dawn, B. (2012). Adult bone marrow cell therapy improves survival and induces long-term improvement in cardiac parameters/clinical perspective. Circulation, 126, 551-568. doi:10. 1161/ CIRCULATIONAHA.111.086074.

5. van Ramshorst, J., Bax, J. J., Beeres, S. L., et al. (2009). Intramyocardial bone marrow cell injection for chronic myocardial ischemia: a randomized controlled trial. JAMA, 301, 1997-2004. doi:10.1001/jama.2009.685.

6. Perin, E. C., Silva, G. V., Assad, J. A., et al. (2008). Comparison of intracoronary and transendocardial delivery of allogeneic mesenchymal cells in a canine model of acute myocardial infarction. Journal of Molecular and Cellular Cardiology, 44, 486-495. doi: 10.1016/j.yjmcc.2007.09.012.

7. Van der Spoel, T. I. G., Vrijsen, K. R., Koudstaal, S., et al. (2012). Transendocardial cell injection is not superior to intracoronary infusion in a porcine model of ischemic cardiomyopathy: a study on delivery efficiency. Journal of Cellular and Molecular Medicine, 16, 2768-2776. doi:10.1111/j.1582-4934.2012.01594.x.

8. Timmers, L., Henriques, J. P. S., de Kleijn, D. P. V., et al. (2009). Exenatide reduces infarct size and improves cardiac function in a porcine model of ischemia and reperfusion injury. Journal of the American College of Cardiology, 53, 501-510. doi:10.1016/j.jacc. 2008.10.033.

9. Noort, W. A., Oerlemans, M. I. F. J., Rozemuller, H., et al. (2012). Human versus porcine mesenchymal stromal cells: phenotype, differentiation potential, immunomodulation and cardiac improvement after transplantation. Journal of Cellular and Molecular Medicine, 16, 1827-1839. doi:10.1111/j.1582-4934.2011.01455.x.

10. Ben-Haim, S. A., Osadchy, D., Schuster, I., Gepstein, L., Hayam, G., \& Josephson, M. E. (1996). Nonfluoroscopic, in vivo navigation and mapping technology. Nature Medicine, 2, 1393-1395.

11. Gepstein, L., Hayam, G., \& Ben-Haim, S. A. (1997). A novel method for nonfluoroscopic catheter-based electroanatomical mapping of the heart: in vitro and in vivo accuracy results. Circulation, 95, 1611-1622. doi:10.1161/01.CIR.95.6.1611.

12. Baan, J., van der Velde, E. T., de Bruin, H. G., Smeenk, G. J., Koops, J., van Dijk, A. D., Temmerman, D., Senden, J., \& Buis, B. (1984). Continuous measurement of left ventricular volume in animals and humans by conductance catheter. Circulation, 70, 812823. doi:10.1161/01.CIR.95.6.1611.

13. Steendijk, P., \& Baan, J. (2000). Comparison of intravenous and pulmonary artery injections of hypertonic saline for the assessment of conductance catheter parallel conductance. Cardiovascular Research, 46, 82-89.

14. Steendijk, P., Baan, J., Jr., Van Der Velde, E. T., \& Baan, J. (1998). Effects of critical coronary stenosis on global systolic left ventricular function quantified by pressure-volume relations during 
dobutamine stress in the canine heart. Journal of the American College of Cardiology, 32, 816-826. doi:10.1016/S0735-1097(98) 00313-1.

15. Ten Brinke, E. A., Klautz, R. J., Verwey, H. F., van der Wall, E. E., Dion, R. A., \& Steendijk, P. (2010). Single-beat estimation of the left ventricular end-systolic pressure-volume relationship in patients with heart failure. Acta Physiologica, 198, 37-46. doi:10.1111/j. 1748-1716.2009.02040.

16. Sluijter, J. P. G., Smeets, M. B., Velema, E., Pasterkamp, G., \& de Kleijn, D. P. V. (2004). Increased collagen turnover is only partly associated with collagen fiber deposition in the arterial response to injury. Cardiovascular Research, 61, 186-195.

17. Li, S. R., Qi, X. Y., Hu, F. L., et al. (2008). Mechanisms of improvement of left ventricle remodeling by trans-planting two kinds of autologous bone marrow stem cells in pigs. Chinese Medical Journal, 121, 2403-2409.

18. Schachinger, V., Erbs, S., Elsasser, A., et al. (2006). Intracoronary bone marrow-derived progenitor cells in acute myocardial infarction. New England Journal of Medicine, 355, 1210-1221. doi:10. 1056/ NEJMoa060186.

19. Huikuri, H. V., Kervinen, K., Niemela, M., et al. (2008). Effects of intracoronary injection of mononuclear bone marrow cells on left ventricular function, arrhythmia risk profile, and restenosis after thrombolytic therapy of acute myocardial infarction. European Heart Journal, 29, 2723-2732. doi:10.1093/eurheartj/ehn436.

20. Cao, F., Sun, D., Li, C., et al. (2009). Long-term myocardial functional improvement after autologous bone marrow mononuclear cells transplantation in patients with ST-segment elevation myocardial infarction: 4 years follow-up. European Heart Journal, 30, 1986-1994. doi:10.1093/ eurheartj /ehp220.

21. Perin, E. C., Willerson, J. T., Pepine, C. J., et al. (2012). Effect of transendocardial delivery of autologous bone marrow mononuclear cells on functional capacity, left ventricular function, and perfusion in chronic heart failure: the FOCUS-CCTRN trial. JAMA, 307, 1717-1726. doi:10.1001/ jama.2012.418.

22. Santoso, T., Siu, C. W., Irawan, C., et al. (2014). Endomyocardial implantation of autologous bone marrow mononuclear cells in advanced ischemic heart failure: a randomized placebo-controlled trial (END-HF). Journal of Cardiovascular Translational Research, 7, 545-552. doi:10.1007/s12265-014-9580- 6.
23. Williams, A. R., Trachtenberg, B., Velazquez, D. L., et al. (2011). Intramyocardial stem cell injection in patients with ischemic cardiomyopathy/novelty and significance. Circulation Research, 108, 792-796. doi:10.1161/CIRCRESAHA.111.242610.

24. Heldman, A. W., DiFede, D. L., Fishman, J. E., et al. (2014). Transendocardial mesenchymal stem cells and mononuclear bone marrow cells for ischemic cardiomyopathy: the TAC-HFT randomized trial. JAMA, 311, 62-73. doi:10.1001/jama.2013.282909.

25. Poh, K. K., Sperry, E., Young, R. G., Freyman, T., Barringhaus, K. G., \& Thompson, C. A. (2007). Repeated direct endomyocardial transplantation of allogeneic mesenchymal stem cells: safety of a high dose, "off-the-shelf", cellular cardiomyoplasty strategy. International Journal of Cardiology, 117, 360-364. doi:10.1016/j. ijcard.2006.04.092.

26. Diederichsen, A. C. P., Moller, J. E., Thayssen, P., Videbaek, L., Saekmose, S. G., Barington, T., \& Kassem, M. (2010). Changes in left ventricular filling patterns after repeated injection of autologous bone marrow cells in heart failure patients. Scandinavian Cardiovascular Journal, 44, 139-145. doi:10.3109/ 14017430903556294.

27. Yao, K., Huang, R., Sun, A., et al. (2009). Repeated autologous bone marrow mononuclear cell therapy in patients with large myocardial infarction. European Journal of Heart Failure, 11, 691-698. doi:10.1093/eurjhf/hfp062.

28. Gavira, J. J., Nasarre, E., Abizanda, G., et al. (2010). Repeated implantation of skeletal myoblast in a swine model of chronic myocardial infarction. European Heart Journal, 31, 1013-1021. doi:10. 1093/eurheartj/ehp342.

29. Siegel, G., Krause, P., Wöhrle, S., et al. (2012). Bone marrowderived human mesenchymal stem cells express cardiomyogenic proteins but do not exhibit functional cardiomyogenic differentiation potential. Stem Cells and Development, 21, 2457-2470. doi:10. 1089/scd.2011.0626.

30. Seeger, F. H., Rasper, T., Bönig, H., et al. (2014). The challenges of autologous cell therapy: systemic anti-thrombotic therapies interfering with serum coagulation may disable autologous serumcontaining cell products for therapeutical use. Journal of Cardiovascular Translational Research, 7, 644-650. doi:10.1007/ s12265-014-9584-2. 